\title{
Electric field in two-dimensional complex plasma crystal: Ideal lattices
}

\author{
B. BAHADORY*, R. MOHAZABYeH \\ ${ }^{a}$ Atomic and Molecular Department, Physics Faculty, Yazd University, Yazd 8915818411, Iran \\ (Received August 06, 2019; revised version September 03, 2019; in final form September 11, 2019) \\ Two-dimensional complex plasma crystal is a suspended single-layer of micron-size grains in a plasma. The nar- \\ row and long two-row crystals are likely to be used as electrostatic wigglers. Therefore, electric properties \\ of such system is studied in the present work. The frequency of longitudinal electric field is twice the frequency \\ of transverse electric field. In two-row crystals, far from the crystal center, both longitudinal and transverse \\ electric fields decrease continuously. Frequency of longitudinal field decreases also, but the frequency of trans- \\ verse field is fix. By increasing distance between the crystal rows, the frequency of transverse field does not \\ change but the frequency of longitudinal field decreases. Value of both fields decrease while their ratio increases. \\ By increasing longitudinal distance between the grains, the frequency of transverse field does not change but \\ the frequency of longitudinal field increases until become twice the frequency of transverse field, and both electric \\ fields increase while their ratio decreases. These results may be useful in various fields of physics.
}

DOI: 10.12693/APhysPolA.136.968

PACS/topics: complex plasma, plasma crystal, free-electron laser

\section{Introduction}

Complex (dusty) plasma is a plasma that contains grains (particles). In an ordinary experiment, grains are spherical with order of micrometer in size [1,2]. First observation of crystal formation in a plasma environment was done by independent groups in 1994 [3, 4]. Complex plasmas have been typically generated in parallel plate radio-frequency discharge. Grains introduced into the plasma chamber become electrically charged. Due to faster mobility of electrons, grains obtain negative charge. Those grains with high charge and low temperature form a complex plasma crystal $[5,6]$. To measure stability of the plasma crystals, Coulomb coupling parameter is used $[7,8]$ :

$$
\Gamma=\frac{k q^{2}}{d k_{\mathrm{B}} T} \exp \left(-d / \lambda_{\mathrm{D}}\right)
$$

where $k$ is the Coulomb's constant, $q$ is grains charge, $d$ is intergrain distance, $k_{\mathrm{B}}$ is the Boltzmann constant, $T$ is grains temperature, and $\lambda_{\mathrm{D}}$ is Debye length of the system. Complex plasma crystals are twodimensional (2D) usually. The reason is balancing between downward gravitational force and upward electric field generated by lower electrode $[9,10]$.

The complex plasma crystals are suitable for fundamental researches because they are formed easily, and because of direct optical imaging of individual particles with large size and large inter-spacing. This sort of crystals has applications in different fields from nanotechnology to plasma physics and free-electron lasers,

\footnotetext{
*corresponding author; e-mail: bahadory@netc.fr
}

FELs $[1,11]$. More examples include also in trapped ions; few-body systems; formation, melting and phase transition of crystals; asymmetric crystals and crystal defects; waves; and non-ideal plasmas.

Microstructure electrostatic wigglers is a new possible application of complex plasma crystals. It is suggested by Mirzanejhad and Bahadory [12]. Electrostatic wigglers can transfer energy to relativistic electron beam in FELs. It is an advantage and can lead to a more powerful laser. Various electrostatic wigglers are studied, e.g., metal and sinusoidal shape in [13, 14], while electrodes with oscillatory voltages in [15].

In the microstructure electrostatic wiggler, charge and consequentially potential and electric field of individual grains are important [12]. Average grain charge has been already reported, but there are no experimental measurements of individual grains charge. 'The 2012 Plasma Roadmap' [16] has refered to this problem and stated that developing novel probes to study charge distributions of grains in plasmas would be of great benefit. The problem still exists and so far new studies consider only the external electric fields [17-20].

We have simulated $2 \mathrm{D}$ plasma crystals with different shapes in previous work [21]. One category of shapes, meaning long and narrow two-row crystals, has been especially suitable as electrostatic wiggler. In this work, we consider complex plasma with these type of crystals and investigate electric fields of grains in detail. We find shape and value of electric fields in different area of the crystals, as well as for different grains distances. In all kind of electrostatic wigglers, electric fields must be determined. So, our results can be used in designing and production of FELs specially, but they may be useful in other fields as well (for example in solid-state physics). 
The perfect crystal without any vacancies are subject of study of the present work. It is hard to produce ideal crystals, however they are the reference base when one needs to verify results of simulated and of real crystals. Vacancy and any other disordering leading to non-ideal crystals, will be a subject of our ongoing work.

\section{Electric field of one grain}

As mentioned in Introduction, electric field of individual grains are important. Grains potential energy also plays an important role in crystal formation. With assumption of the screened Coulomb (DebyeHückel) potential for a grain one can write potential for a grain as $[22,23]$ :

$$
U=\frac{k q}{r} \exp \left(-r / \lambda_{\mathrm{D}}\right)
$$

where $r$ is distance from the grain. The electric field of single grain becomes

$$
E_{\text {grain }}=\frac{k q}{r^{2}}\left(1+\frac{r}{\lambda_{\mathrm{D}}}\right) \exp \left(-r / \lambda_{\mathrm{D}}\right) .
$$

Because of the Debye shield, the electric field in complex plasma is always less than vacuum environment. Figure 1 shows that the electric field of one grain in complex plasma, $E_{\text {grain }}$, is less than the Coulomb electric field of one grain in vacuum, $E_{\text {coulomb. }}$. As expect, both fields vanish in faraway.

As can be seen in Fig. 1, the change in the grain charge has no effect on behavior of the electric fields. This has been done and tested, e.g., by doubling the amount of charge, one has doubled the amount of the electric field, while the shape of the electric field is unchanged. So, for all calculations in this paper, we get arbitrary but typical amount of the grain charge to be $q=10000 e[24,25]$.

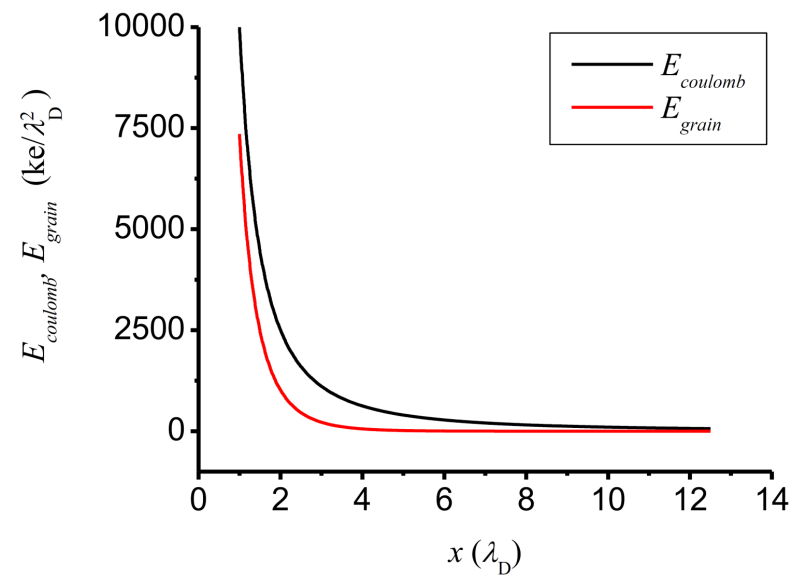

Fig. 1. Comparison of coulomb electric field in vacuum, $E_{\text {coulomb }}$, and in complex plasma, $E_{\text {grain }}$. Source charge is a grain at $x=0$ and $y=0$, and observation line is at $y=0$.

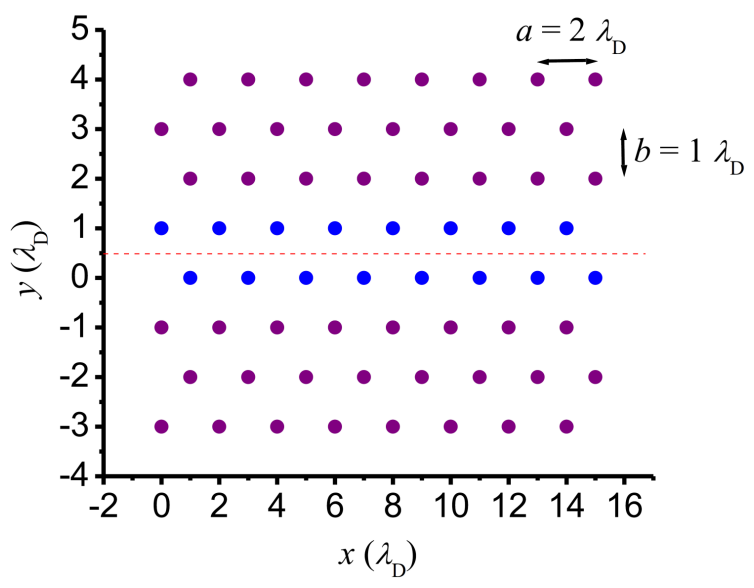

Fig. 2. Two-dimensional ideal lattice in complex plasma. Blue colors show a two-row crystal. Observation line is plotted in red dash. Distance between any two row is $b=1 \lambda_{\mathrm{D}}$ and distance between any two successive grains in each row is $a=2 \lambda_{\mathrm{D}}$.

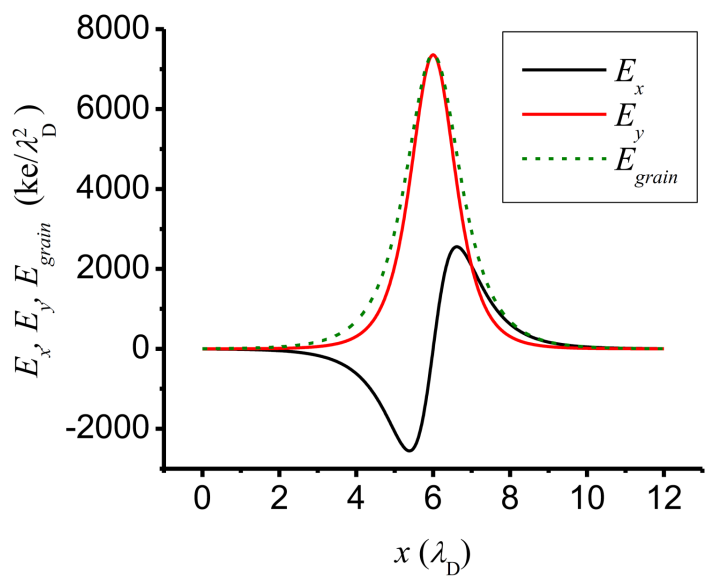

Fig. 3. The longitudinal, transvers and total electric fields of one grain. The grain is located at $x=6 \lambda_{\mathrm{D}}$ and $y=1 \lambda_{\mathrm{D}}$, and observation line is located at $y=2 \lambda_{\mathrm{D}}$.

Our system, shown in Fig. 2, is two-dimensional. We call the $x$ and $y$ components of the electric fields as longitudinal and transverse components of the electric fields, respectively, $E_{x}$ and $E_{y}$. To determine the net electric fields inside the crystal, the electric field of single grain is calculated at first. Then, by using the superposition principle, the electric fields of all grains is determined. Consider now single grain located at $x=6 \lambda_{\mathrm{D}}$ and $y=1 \lambda_{\mathrm{D}}$, and ignore other grains. The observation line is chosen at $y=2 \lambda_{\mathrm{D}}$. In Fig. 3 one can see the electric field and its components in case of considered grain. At the grain location, i.e., $x=6 \lambda_{\mathrm{D}}$, the component $E_{y}$ has maximum value and the component $E_{x}$ is zero. Further, there are the two oppositely directed peaks of $E_{x}$ for one peak of $E_{y}$. Away from the grain location, $E_{x}$ and $E_{y}$ vanish exponentially. Other figures of this paper are superposition of Fig. 3. 


\section{Electric field in a two-row crystal}

We define the following configuration as an ideal lattice: each grain arranges in the middle of two grains of top row and two grains of bottom row (see Fig. 2). The arbitrary lattice consists of 8 rows with each row contains 8 grains. By keeping two rows in the middle of the lattice and omitting other rows, one obtains a special kind of crystals forms: two-row crystal. Blue color in Fig. 2 shows a two-row crystal. The properties of tworow crystals were simulated and analyzed in [21].

The longitudinal and transverse electric fields, $E_{x}$ and $E_{y}$ respectively, in middle of the two-row crystal in that each row containing 8 grains are shown in Fig. 4 . Observation line is marked by red dash line in Fig. 2 and as $x$-axis in Fig. $4 \mathrm{~b}$. Figures $4 \mathrm{a}$ and $4 \mathrm{~b}$ are the same but they have different scale. In addition, Fig. $4 \mathrm{~b}$ includes the grains. Distance between two-row is $b=1 \lambda_{\mathrm{D}}$ and distance between any two successive grains in each row is $a=2 \lambda_{\mathrm{D}}$ (see Fig. 2). These are characteristic parameters. Other parameters will be used in the next section.

In ideal two-row crystals, as Fig. 4 shows, number of $E_{y}$ peaks is equal to number of grains, and number of $E_{x}$ peaks is twice the number of $E_{y}$ peaks. Accordingly, the frequency of electric field in the longitudinal direction is twice the frequency in the transverse direction. Peaks of $E_{y}$ occur at grains position $x$, where $E_{x}$ is zero. Importantly, between two successive grains both fields are zero. We will now use notation $E_{x \text {,max }}$ and $E_{y \text {,max }}$ to indicate maximum value of $E_{x}$ and $E_{y}$, respectively. At any position $x$ that $E_{x}$ has maximum value, the ratio $E_{y, \max } / E_{x, \max }$ is constant and equals $\sim 4.47$. In Fig. 4a, one can see also effect of crystal boundaries at the beggining and the ending.

If we consider 4 rows of grains or more, such like in Fig. 2, the electric fields behavior does not change, however, its quantity change a bit. For example, the electric fields for ten rows, where each row contains 8 grains, was computed. Component $E_{x}$ and $E_{y}$ have 32 and 16 peaks respectively. When the number of rows in the lattice increases from two to ten, $E_{x, \max }$ increases about $0.1 \%, E_{y, \max }$ decreases about $1.7 \%$, and $E_{y, \max } / E_{x, \max }$ ratio decreases about $1.9 \%$. Results of other cases are similar to this one. Thus one can conclude that other rows of lattices have negligible effect. In the rest part of this paper we will study two-row crystals with 8 grains in each row only.

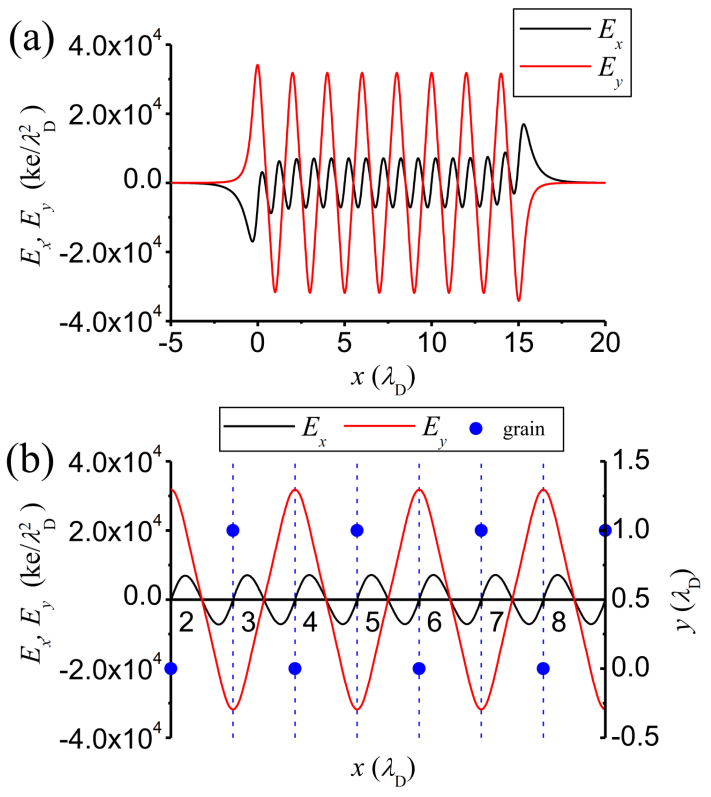

Fig. 4. (a) The longitudinal and transverse electric fields in the middle of the two-row crystal. (b) Magnified middle part of panel (a) in addition to the grains location.
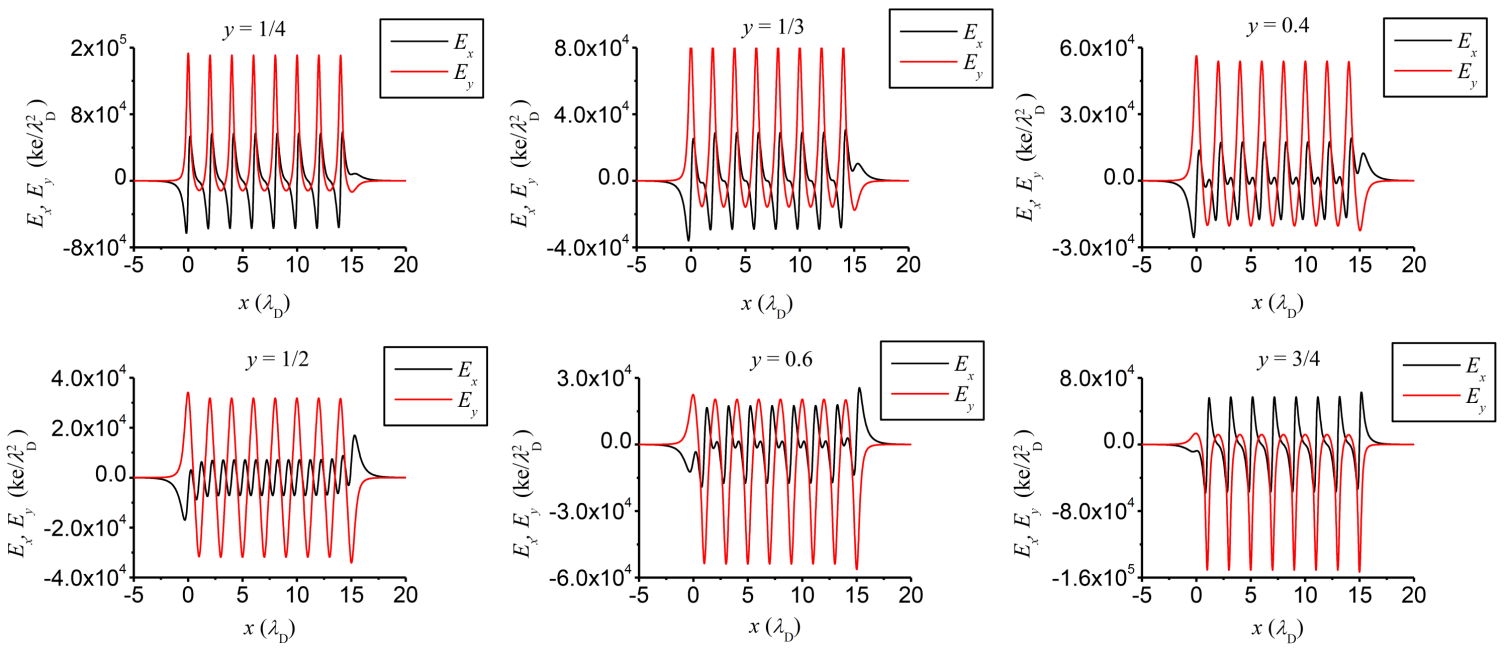

Fig. 5. The longitudinal and transverse electric fields in different areas of the two-row crystal. The rows are located at $y=0$ and $y=1 \lambda_{\mathrm{D}}$, and each row contains 8 grains. 

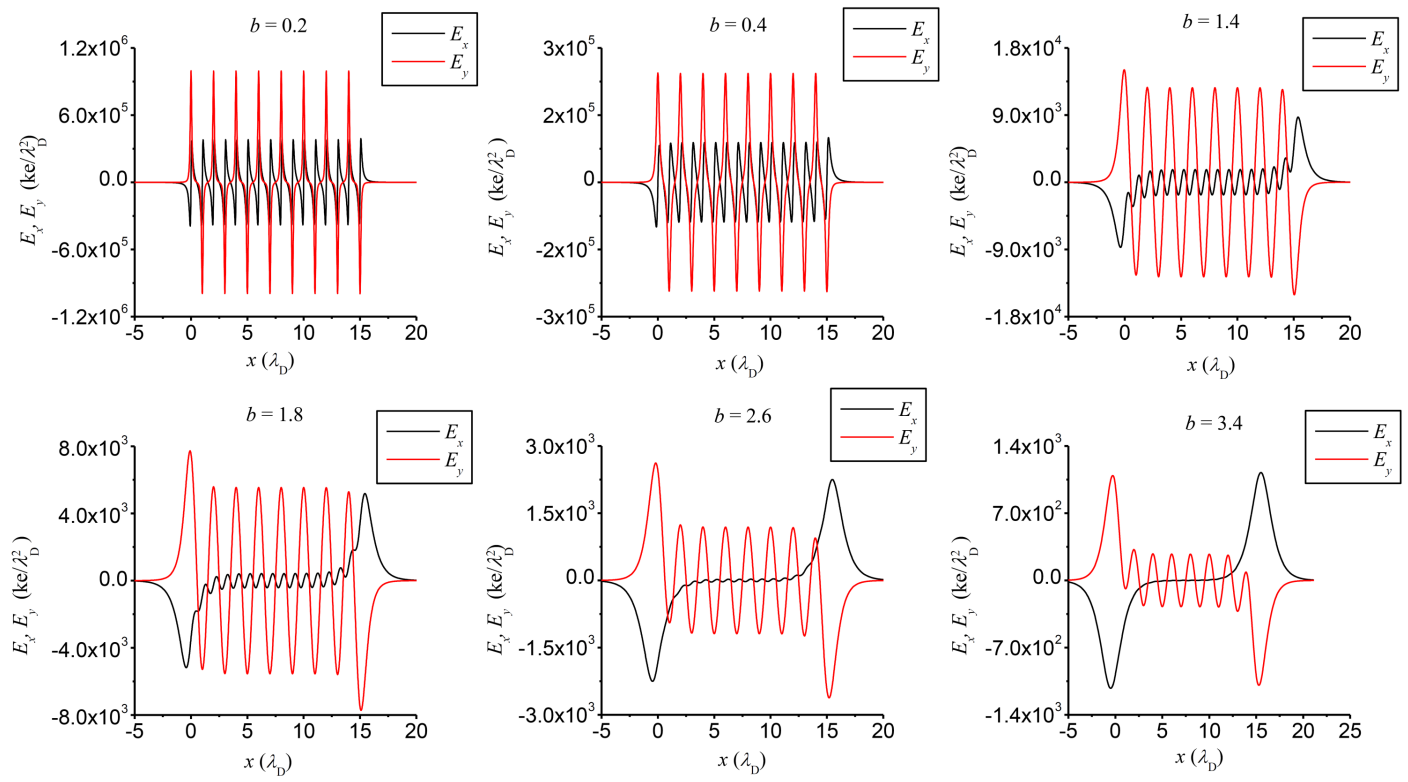

Fig. 6. Effect of transverse distance between the grains, $b$, on the electric fields of the complex plasma crystal. By increasing $b$, the peaks height of both electric fields decreases, the number of $E_{y}$ peaks does not change, and the number of $E_{x}$ peaks decreases.

Out of the middle of the two-raw crystal, both electric fields components and their values, change. We move observation line step by step from the middle to the edges of the crystal. Figure 5 contains main results. $E_{x}$ at $y=1 / 4 \lambda_{D}$ is similar to $E_{x}$ at $y=3 / 4 \lambda_{\mathrm{D}}$, while the components $E_{y}$ at $y=1 / 4 \lambda_{D}$ and $y=3 / 4 \lambda$ have opposite directions. In both cases at observation line possition, the $E_{x}$ and $E_{y}$ have 16 peaks. In case when $y=1 / 3 \lambda_{\mathrm{D}}$ a little flat line appears between each two successive $E_{x}$ peaks. In the range from $y=0.4 \lambda_{\mathrm{D}}$ to $y=0.6 \lambda_{\mathrm{D}}$, peaks of $E_{x}$ start to doubling from 16 to 32 . This process complete in $y=1 / 2 \lambda_{\mathrm{D}}$. (Figure 4 is identical to case $y=1 / 2 \lambda_{\mathrm{D}}$ in Fig. 5). The behavioral changes occur gradually and with complexity. Though, closer to the crystal center, both $E_{x}$ and $E_{y}$ decrease continuously.

\section{Effect of grains distance}

In the previous section we focused on the electric fields at different positions in the two-row crystal. The observation line is set exactly in the middle of the two-row crystal. We will now examine the effect of longitudinal and transverse distance between grains $a$ and $b$ (see Fig. 2.).

At first, we select $a=2 \lambda_{\mathrm{D}}$ as a constant and change distance $b$ step by step. Figure 6 shows results for $b=0.2,0.4,1.4,1.8,2.6$ and $3.4 \lambda_{\mathrm{D}}$. Generally, by increasing $b$ peaks height of both electric fields decreases. The number of $E_{y}$ peaks does not change, and the number of $E_{x}$ peaks decrease for a certain $b$ value. For example, at the minimal value of $b=0.2 \lambda_{\mathrm{D}}$, both $E_{x}$ and $E_{y}$ are maximal relative to other cases. From $b=0.2 \lambda_{\mathrm{D}}$ to $b=1.4 \lambda_{\mathrm{D}}$, the number of $E_{y}$ peaks is equal to the number of grains, which is 16 , and the number of $E_{x}$

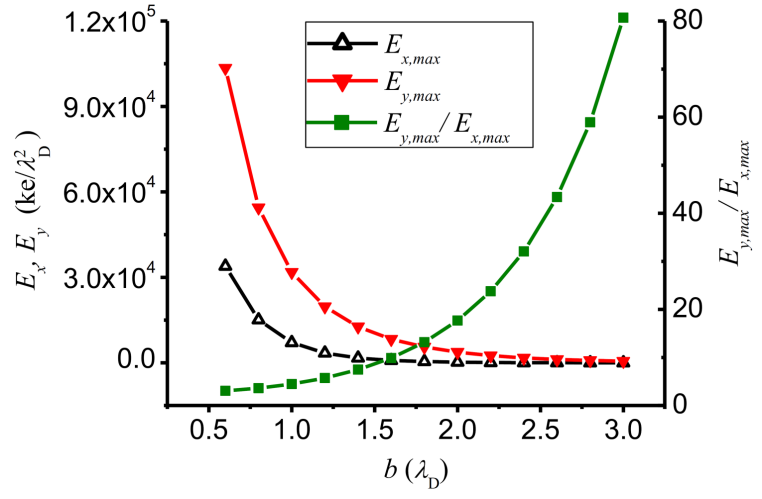

Fig. 7. The longitudinal and transverse electric fields and their ratio as function of transverse distance between the grains, $b$.

peaks is twice them, that is 32 . Starting from $b=1.8 \lambda_{\mathrm{D}}$, $E_{x}$ peaks start to decrease, so that for $b=2.6 \lambda_{\mathrm{D}}$ the number of $E_{x}$ peaks is 18. At bigger distances, the electric fields vanish gradually. Exception are only the crystal boundaries. $E_{x, \max }, E_{y, \max }$ and their ratio as a function of $b$ are plotted in Fig. 7. Both electric fields decrease when increasing $b$, while their ratio increases.

Now, we set transvers distance between the rows at $b=1 \lambda_{\mathrm{D}}$ and change $a$ step by step. Figure 8 shows results for $a=0.6,0.8,1.2,2.0,5.0$ and $10.0 \lambda_{\mathrm{D}}$. Note that for longitudinal distance $a=0.6 \lambda_{\mathrm{D}}$, the lattice becomes small. One can observed then only 2 peaks of $E_{x}$ at beginning and ending, and 16 peaks of $E_{y}$. In $a=$ $0.8 \lambda_{\mathrm{D}}$, the effect of inner grains appears. In $a=1.2 \lambda_{\mathrm{D}}$, 

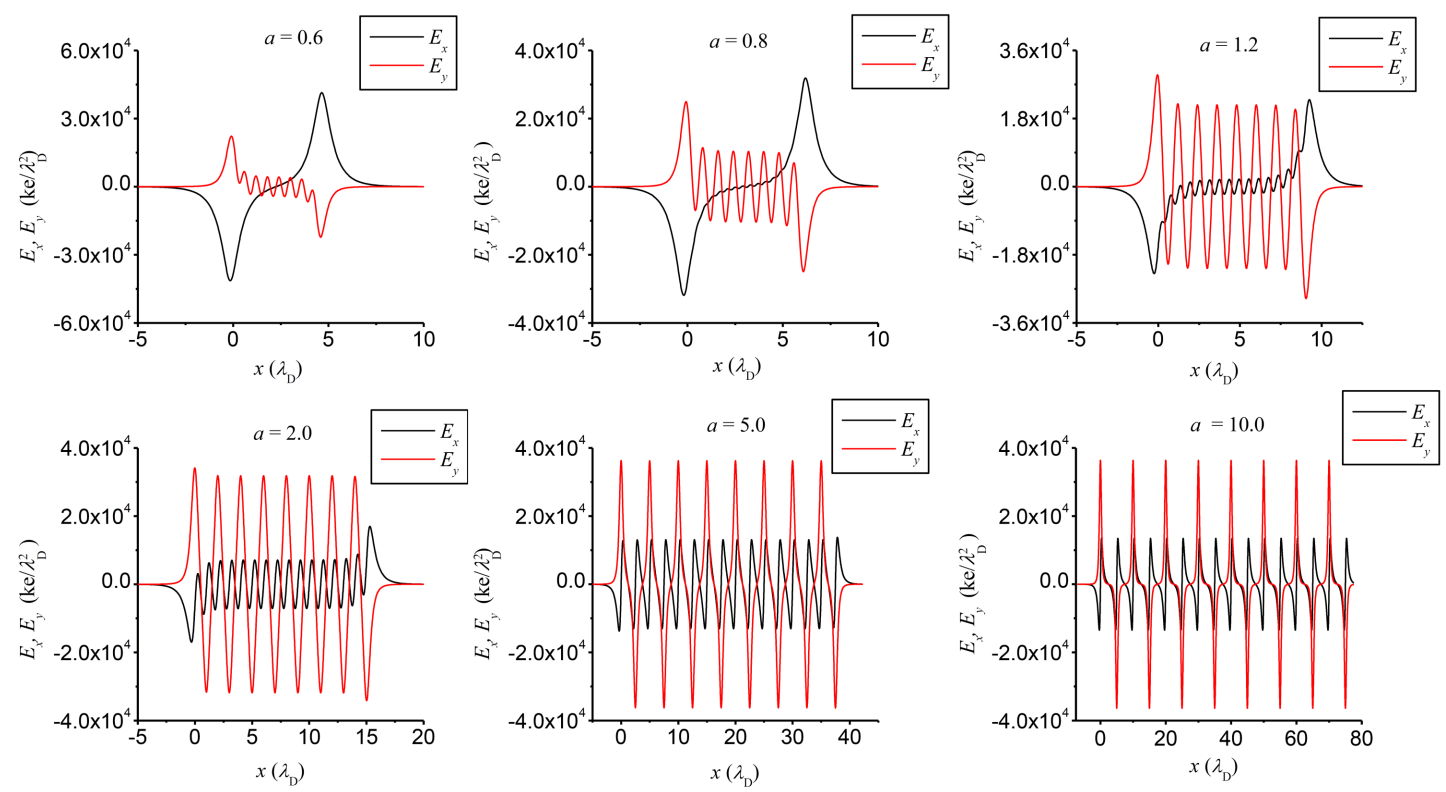

Fig. 8. Effect of longitudinal distance between grains, $a$, on the electric fields of the complex plasma crystal. By increasing $a$, the peaks height of both electric fields increases, the number of $E_{y}$ peaks does not change, and the number of $E_{x}$ peaks increases.

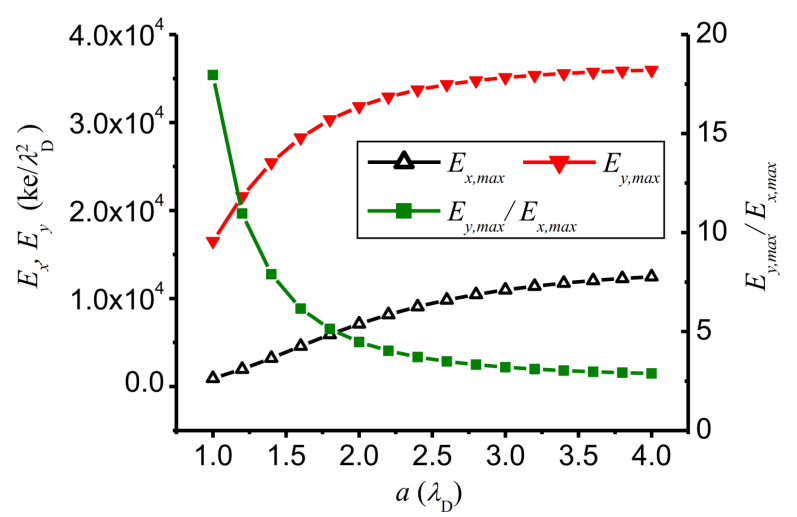

Fig. 9. The longitudinal and transverse electric fields and their ratio as function of longitudinal distance between the grains, $a$.

the individual effect of each grain forms (but not completely) while the number of $E_{x}$ peaks is less than in normal case. This process completes in $a=1.4 \lambda_{\mathrm{D}}$, so that the number of $E_{x}$ peaks becomes 32 and this is twice the $E_{y}$ peaks and twice the grains number (we plotted $a=2.0 \lambda_{\mathrm{D}}$ for more clarity). Generally, by increasing $a$, the peaks height of both electric fields components increase. The number of $E_{y}$ peaks does not change, and the number of $E_{x}$ peaks increases until a certain $a$. In $a=10 \lambda_{\mathrm{D}}$ the electric fields of grains, have not overlap. $E_{x, \max }, E_{y, \max }$, and their ratio as function of $a$ are plotted in Fig. 9. Both electric fields increase when $a$ increases, while their ratio decreases. In fact, all results concerned constant $b$ are the opposite with the case for constant $a$.

\section{Conclusion}

The electric field is one of the fundamental properties of the complex plasma crystals. In this paper, we analyzed the electric field of single grain. It was shown that the frequency of the longitudinal electric field is twice the frequency of the transverse electric field. In near areas to grains, the transverse electric field is stronger than the longitudinal electric field. $E_{x}$ is zero at grains position $x$, while $E_{y}$ is maximum at the same points.

We study also the effect of row numbers on electric field behaviour. Our observations indicate that large number of rows have a little effect on the longitudinal and transverse fields, and the first two rows have main effects in fact. By increasing the number of rows in the lattice from 2 to 10, peaks of $E_{x}$ increases, but peaks of $E_{y}$ and ratio $E_{y, \max } / E_{x, \max }$ decrease (less than $2 \%$ ).

In the two-row crystals, away from its center, both $E_{x}$ and $E_{y}$ decrease continuously. Frequency of $E_{x}$ decreases while frequency of $E_{y}$ is fix. Due to this, the fields asymmetry occurs gradually and with complexity.

Longitudinal and transverse distances between grains have essential role in quality and value of the fields. For constant longitudinal distance between grains, the increase of transverse distance between rows does not change $E_{y}$ frequency but decreases the $E_{x}$ frequency. The value of $E_{x}$ and $E_{y}$ in turn decreases, while their ratio increases. For constant transverse distance between grains, by increasing longitudinal distance between grains, both electric fields increase, while their ratio decreases, $E_{y}$ frequency does not change, and $E_{x}$ frequency increases until become twice $E_{y}$ frequency. 
In addition to cold plasma physics, the results of the presented work may be useful for some other research fields such as Wigner crystals and 2D materials. Because of quantifying of the electric fields, the results are also useful in design and production of microstructure electrostatic wigglers.

\section{References}

[1] S.V. Vladimirov, K. Ostrikov, and A.A Samarian, Physics and applications of complex plasmas, Imperial College Press, London 2005.

[2] V.N. Tsytovich, G. Morfill, S.V. Vladimirov, and H.M. Thomas, Elementary physics of complex plasmas, Springer Science \& Business Media, Berlin 2008.

[3] J.H. Chu, I. Lin, Phys. Rev. Lett. 72, 4009 (1994).

[4] H. Thomas, G.E. Morfill, V. Demmel, J. Goree, B. Feuerbacher, D. Möhlmann, Phys. Rev. Lett. 73 652 (1994).

[5] J. Yan, F. Feng, F.C. Liu, Y.F. He, Chin. Phys. B 26, 095202 (2017).

[6] V.E. Fortov, A.V. Ivlev, S.A. Khrapak, A.G. Khrapak, G.E. Morfill, Phys. Rep. 421, 1 (2005).

[7] O. Ishihara, J. Phys. D: Appl. Phys. 40, R121 (2007).

[8] Z. Donkó, P. Hartmann, P.K. Shukla, Phys. Lett. A 376, 3199 (2012).

[9] W.T. Juan, Z.H. Huang, J.W. Hsu, Y.J. Lai, I. Lin, Phys. Rev. E 58, R6947 (1998).
[10] T.B. Röcker, A.V. Ivlev, S.K. Zhdanov, L. Couëdel, G.E. Morfill, Phys. Plasmas 21, 073711 (2014).

[11] B. Bahadory, S. Mirzanejhad, Phys. Scr. 84, 035501 (2011).

[12] S. Mirzanejhad, B. Bahadory, Phys. Plasmas 14, 043101 (2007)

[13] S. Zhang, Phys. Rev. ST Accel. Beams 13, 090701 (2010).

[14] N. Sepehri Javan, Phys. Plasmas 17, 063105 (2010).

[15] M. Nikrah, S. Jafari, Laser Phys. 26, 065005 (2016).

[16] S. Samukawa, et al. J. Phys. D: Appl. Phys. 45, 253001 (2012).

[17] W. Kim, P.V. Pikhitsa, M. Choi, Appl. Phys. Lett. 109, 193107 (2016).

[18] G.I. Sukhinin, A.V. Fedoseev, M.V. Salnikov, A. Rostom, M.M. Vasiliev, O.F. Petrov, Phys. Rev. E 95, 063207 (2017).

[19] I. Lisina, E. Lisin, O. Vaulina, Phys. Plasmas 23 033704 (2016).

[20] Y. Saitou, Phys. Plasmas 25, 073701 (2018).

[21] B. Bahadory, Chin. Phys. B 27, 025202 (2018).

[22] K. Avinash, Phys. Rev. Lett. 98, 095003 (2007).

[23] O.S. Vaulina, S.V. Vladimirov, O.F. Petrov, V.E. Fortov, Phys. Rev. Lett. 88, 245002 (2002).

[24] V.A. Schweigert, I.V. Schweigert, V. Nosenko, J. Goree, Phys. Plasmas 9, 4465 (2002).

[25] S.K. Sharma, R. Kalita, Y. Nakamura, H. Bailung, Plasma Sources Sci. Technol. 21, 045002 (2012). 